\title{
REVIEW PAPERS
}


Alan H. Batten

Dominion Astrophysical Observatory

Herzberg Institute of Astrophysics

\begin{abstract}
Standard stars should normally be constant in the characteristic for which they have been chosen to be standards. Individually they should be capable of testing the instrument used in their measurement, and collectively (usually together with a particular type of instrument) they must define a system of measurement. A star should not be adopted as a standard until several years' observation have demonstrated its constancy. The number of independent observations in that interval is as important as its length. Many of these points are illustrated by particular reference to standard-velocity stars. Even 100 observations of such a star may fail to reveal a detectable variation that is present. A distinction is drawn between "primary" standards, meeting the above criteria, and "reference" or "comparison" stars which need not be so severely tested since their use is more limited. Standard stars must be calibrated in some fundamental way. Since random errors of observation can introduce systematic errors in calibration, the use of the intermediate step of standard stars may decline with increasing internal precision of observations.
\end{abstract}

\title{
1. INTRODUCTION
}

The theme of this symposium is the calibration of astronomical measurements, especially of those that lead to knowledge of the fundamental stellar quantities of mass, radius, luminosity, temperature, etc. We wish to take stock of the uncertainties, both accidental and systematic, that may yet remain in our knowledge of these quantities; not only because stars themselves are interesting, but because our knowledge of the whole universe depends on how accurately we know these fundamental things about them. In one respect, even Copernicus could not displace the Earth from the center of our Universe. Although we have begun to observe from space, most of us are still bound to the Earth and, both historically and logically, have had to explore the Universe outwards from our home. There is a chain of inference connecting our first measurements of the size and shape of the Earth, through the determination of the dimensions of the Solar System and the 
parallaxes of stars, to our modern claim to know, at least approximately, the size and "age" of the Universe. Many of us would like to examine this whole chain, but we have been persuaded that that would be impracticable in one symposium. Our attention, therefore, is concentrated on a few links in the middle of the chain, and you are here because you have devoted much of your astronomical lives to forging those links. We hope, in the next few days, to discharge our responsibility to colleagues working on other links, by inspecting our own very carefully. In our necessarily close and even detailed inspection, however, we should remain aware of the whole chain of inference, and remember our colleagues who also advance our understanding of the Universe, whether their principal interest is closer at home or farther away than ours.

When we measure something about a star - velocity, luminosity, spectral type etc. - we often find it necessary or convenient to do so by comparison with one or more standard stars. Radial velocities are, indeed, measured by comparing the position of features in a stellar spectrum with those in a comparison spectrum that has been measured in the laboratory; but the rest wavelengths appropriate to the stellar features have been determined from the spectra of standard stars whose velocities have been determined (we hope reliably) by our predecessors. In classifying stellar spectra, we rely heavily on standard classifications, made by experts, to estimate - usually fairly easily and quickly - the type of our unknown spectrum. Photometers are usually calibrated by the observation of a set of standard stars rather than the measurement of the output of a standard lamp. The first kind of measurement is an example of a mixture of calibration by laboratory and stellar standards. The second is one of calibration only by standard stars, and it is hard to see how else one could proceed. Unless astronomers one day decide that spectral type is not a useful concept, and refer only to temperatures, surface gravities and abundances, classification is bound to be done by comparison with standards. In the third kind of measurement, there is indeed a clear choice of procedure, but the use of standard stars is overwhelmingly convenient.

\section{WHAT IS A STANDARD STAR?}

The last paragraph makes clear that standards are used and must be chosen for many different purposes. Because of this, we probably all use the term "standard" somewhat loosely at times. Not every star that is used as a reference or comparison is necessarily a standard. We do not always compare an unclassified spectrum directly with one of the standards defining the MK system, choosing instead spectrograms that we may have available of stars that have been reliably classified by comparison with the standards. The light variation of a variable star is usually measured with respect to a comparison star chosen - in order to minimize differential effects - to be close in the sky to the variable and, whenever possible, of similar magnitude and color. The star chosen is most unlikely to be one of the standards defining the photometric system in which the observer is working. The term "standard 
star" must be defined in such a way that we avoid confusion between genuine standards and mere reference or comparison stars. I propose that standards should meet the following requirements:

(i) each individual star should be constant in the characteristic for which it has been chosen as a standard, within the smallest attainable errors of measurement.

(ii) together, the standards for a given characteristic, and the instruments used for their measurement, should define a system.

(iii) individually, the standards should be suitable for testing the performance of the instrument used for their measurement.

The first requirement seems almost too obvious to mention, but it should be the most important in the choice of a standard. Comparison stars for photometry are not always chosen with constancy of light as the sole - or even dominant - consideration. Thus it fairly often happens that a comparison star is itself found to be variable. If the variation is found to be periodic, we probably can salvage the observations. Sometimes we may have no choice but to refer observations of a star under study to a variable of known type. We should, nevertheless, hesitate to call a known spectroscopic binary a velocity standard, or a Cepheid variable a photometric or spectral-classification standard. If all members of a class of stars vary, it may be necessary to adopt some of them as reference stars, but we should not call them standards. Such a situation arises, for example, with M-type giants - all of which vary in light - some of which may be needed as reference stars in a photometric system (McClure 1976).

The second requirement emphasizes that usually we use a set of standards, not just a star. The number of stars in the set will depend, to some extent, on the characteristic being measured and the question will be discussed later. The whole set must be observed in a consistent fashion. Spectral-type standards must be observed at the same dispersion (even with the same spectrograph), with similar emulsions processed in a standard way (Morgan, Keenan and Kellman 1943; Keenan and McNeil 1976.) The UBV system is defined not only by the standard stars, but by the filter, photomultiplier and reflecting surfaces used and even the altitude of the observatory (Johnson and Morgan 1951, 1953; Johnson 1963). Thus a system is defined by a combination of selected stars and carefully specified instruments.

The third requirement underlines that a standard star is something more than a reference against which an unknown star is compared differentially. It can provide a guarantee that instruments are working properly. This is why standards should be observed frequently preferably every night. If a radial-velocity standard consistently gives the "wrong" answer - it being assumed that the star is genuinely a standard in the sense defined and that wavelengths have been carefully selected - not only is at least an approximate "night correction" provided, but the observer is alerted to look for a possible maladjustment in his spectrograph. This is one reason why early-type stars should not be adopted as velocity standards. Even if new techniques of measurement (e.g. cross-correlation) improve the accuracy with which their velocities may be determined, there is no guarantee that any of them are 
sufficiently constant in velocity to be used to detect instrumental errors. Early-type "reference" stars may be very useful - precisely for cross-correlation work, which requires the adoption of such objects for several different sub-types - but, like the M-type giants, they should not be called standards.

\section{CRITERIA FOR THE CHOICE OF STANDARD STARS}

Standard stars are usually chosen by individuals, even when they are approved by a committee. The currently used radial-velocity standards, for example, are largely the choice of Pearce, even though they were formally adopted by I.A.U. Commission 30. The standards defining the MK and UBV systems (Johnson and Morgan 1953) are obviously the work of the originators of these systems and owe their wide acceptance more to their demonstrated usefulness than to any official adoption. Endorsement by an appropriate I.A.U. Commission may be helpful, however, and may be obtained the more readily if definite criteria for the selection of standards are adopted. The most important criterion, reflecting the first requirement in the previous section, is that a star should be constant with respect to the characteristic for which it is proposed as a standard. This criterion can never be fully met. There is always a possibility that an apparently constant star is a long-period variable, or will be revealed as a variable when observations of higher internal precision become possible. Standards must frequently be checked, therefore, and the "state of the art" of observation will limit the choice. In general, the higher the internal precision of our observations, the fewer stars will be acceptable as standards and the harder it will be to find them.

Griffin $(1975,1980)$ recently criticized I.A.U. Commission 30 for what he regarded as too hasty acceptance of a new set of standardvelocity stars. He offered improved values for the velocities of many of the standards and unambiguously demonstrated the variable velocity of one by determining orbital elements for it. He suggested that no star should be adopted as a velocity standard unless its velocity had been shown to be constant for at least ten years. This sounds like a counsel of perfection, but Griffin's strictures were justified and could equally well have been aimed at many of the stars adopted by the same Commission in 1955. Recent studies (Batten et al. 1983, Andersen and Nordström 1983, Mayor unpublished) indicate that several of these standards may have variable velocities, while McClure (1983) has shown that one displays two spectra and is a spectroscopic binary. These discoveries are partly the result of routine observation of the stars in question with higher dispersions than were ordinarily available in the 1950s; but they also reflect that many of the standards were, probably unavoidably, assumed to have constant velocities on insufficient evidence.

Suppose that a star being considered for adoption as a standard for some quantity, $x$, is actually variable in $x$ with a period $\underline{P}$. Suppose also that any deviation from $x_{0}$, the most usual value of $x_{-}^{-}$that is greater than or equal to $\delta x$ can be detected by the observational technique employed. Suppose, finally, that $\underline{x}$ differs from $\underline{x}_{0}$ by at least $\delta \underline{x}$ 
through a fraction $\phi$ of the period; and that the rest of the time $\left|\underline{x}-\underline{x}_{0}\right|<\delta \underline{x}$. These assumptions may seem artificial, but the velocity variation of a spectroscopic binary with an eccentric orbit and longitude of periastron near $0^{\circ}$ or $180^{\circ}$, or the light-variation of an Algo1-type eclipsing system with a very shallow secondary minimum, would nearly satisfy them. Under the assumptions, the probability that one isolated observation would yield a value of $x$ detectably different from $x_{0}$ is simply $\phi$. The probability that it would not do so is $(1-\phi)$. The probability that $\underline{n}$ independent observations would fail to reveal the variation would seem to be

$$
(1-\phi)^{\mathrm{n}}
$$

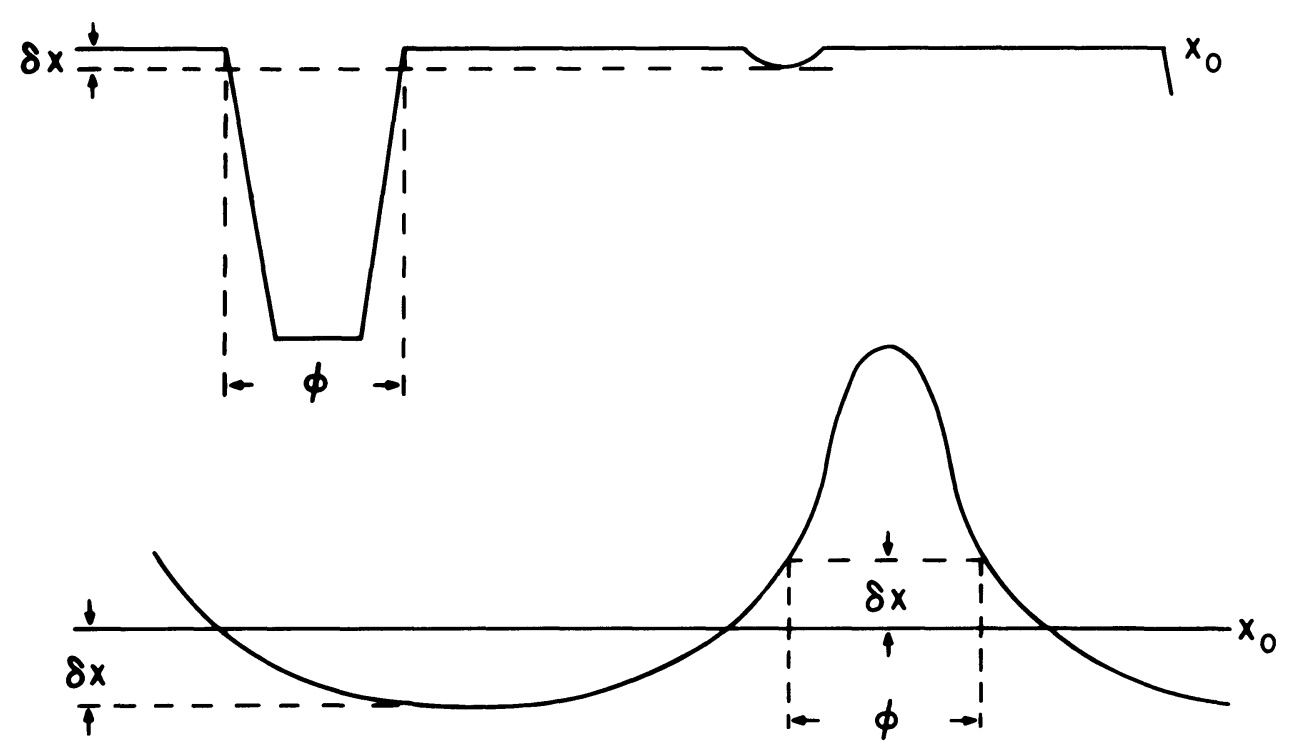

Fig. 1. Schematic light-curve and velocity-curve representing variations that can be discovered only by observations made during a specific interval $\phi$ (expressed as a fraction of the period).

This simple formula gives a lower limit to the probability that an existing variation will not be detected. If the period $P$ is very long, the probability of detection will be diminished. Thus, if $\phi$ is 0.1 and $\underline{P}$ is 10 years, even an infinite number of observations will not reveal the variation unless some are made in the one year out of ten in which it would be detectable; while if $\phi=0.1$ and $P$ is ten months, we would expect to detect the variation after two or three years of serious observation. Particular values of the period (e.g. those close to one year) could also reduce the probability of detecting the variation, as could the finite length of time it takes to make an observation. 
Frequent, regular observation throughout the observing season would increase the probability of detecting a variation, perhaps by more than the formula suggests. Nevertheless, the simple expression $(1-\phi)^{n}$ is a useful first approximation and Table $I$ shows the values it takes for small values of $\phi$ and selected values of $\underline{n}$ :

Table I Probability that n Observations will not detect a variation occuring during a Fraction $\phi$ of a Period $\underline{P}$.

\begin{tabular}{|rlll|}
\hline$\underline{\mathrm{n}} \phi$ & 0.01 & 0.05 & 0.10 \\
\hline 10 & 0.904 & 0.599 & 0.349 \\
50 & 0.605 & 0.077 & 0.005 \\
100 & 0.366 & 0.006 & 0.00003 \\
1,000 & 0.00004 & $5 \times 10^{-23}$ & $2 \times 10^{-46}$ \\
\hline
\end{tabular}

Thus, 1,000 observations will virtually always detect a variation that exists, but 100 observations may quite often miss it and 50 are rarely sufficient to demonstrate constancy.

It is instructive to compare this result with the records for the I.A.U. standard stars listed by Pearce (1957). Of 35 stars fainter than magnitude 4.3 for only two were there more than 30 observations available at the time of selection, and for only nine were there more than 20 observations. The situation was better for the 25 stars brighter than 4.3: only two were selected on the basis of fewer than 20 observations, and for 12 (nearly half) there were 50 observations or more. Nevertheless, $\alpha$ Per - chosen after more than 250 observations - and $\alpha$ Car chosen after nearly 120 - had later to be deleted from the list. I intend no criticism of Pearce's selections by these remarks; he probably made the best choices possible at the time. It is not surprising, however, that new, more accurate observations lead to the rejection, or at least the questioning of many of these standards.

A particularly interesting case history is that of $\mathrm{HD} 184467$, now known to be a binary and first proposed as a standard on the basis of 7 observations. It was about equally likely that these observations would or would not show any variation in velocity, since $\phi \approx 0.1$. (The lowdispersion observations of that time could not have resolved the spectra, but might just have detected a velocity variation.) After another 6 observations had been made, the star was adopted as a standard. There is about one chance in four that 13 observations would fail to reveal the variation. After Petrie and Pearce (1961) had increased the number of observations to about 30 , there was still a four per-cent chance that the variation would escape detection. In fact, they suspected that the adopted velocity needed correction. McClure (1983) was the first to observe the star consistently at higher dispersion and resolved the spectra with his third observation. Although his value of $\delta \underline{x}$ was smaller, and his value of $\phi$ therefore larger, than those of previous observers, he was rather lucky to resolve 
the spectra so early in his own work, although - if all the observations are regarded as one series - his discovery came at just about the stage one might expect.

The frequency with which a prospective standard is observed is as important as the number of years for which it is followed. In an ideal world, I would not only agree with Griffin that prospective standards should be observed for at least ten years, but I would add, they should be observed at least, ten times a year (spread evenly throughout the observing season) from each of three or four observatories. In practice, such a policy would end in no standards ever being accepted, or, at best, being adopted just when techniques of observation have improved to the point at which we must begin the selection and testing all over again. In discussing the frequency of observations, we have to think carefully what we mean by "independent" observations. If our aim is to eliminate the possibility of long-period variations, seven individual observations on consecutive nights are not (apart from the increased precision of their mean) of much more value than one single observation. They cannot, for that purpose, be considered fully independent. Thus, visiting astronomers at national or multi-national observatories can make only a limited contribution to the selection of standards. They should certainly observe standards, both for their own sakes, and to help in the necessarily continual checking. As long as astronomers require standard stars, however, they will need some observatories where the resident staff has first claim on the observing time and where unspectacular long-term programs are encouraged.

The second requirement for standard stars, that they define a system, reminds us that there is no such thing as an isolated standard (except, possibly, the Sun, regarded as the standard for abundance determinations). The stars chosen must cover a range of the quantity for whose measurement they have been selected as standards. Spectraltype standards must cover the whole sequence of spectra. Photometric standards must cover the range of colors and at least be capable of extension to fainter magnitudes. Radial-velocity standards must cover as wide a range of spectral types as is consistent with their being standards. Pearce also wisely chose stars with velocities up to \pm 100 $\mathrm{km} \mathrm{s}^{-1}$. This may not be so important for grating spectrographs as for prism spectrographs, but it is a good principle to follow. There is no unique answer to the question "how many?" standards. Moreover, standards must be spread reasonably unfformly over the sky so that an observer anywhere in the world can have at least some choice on any night of the year. Thus the designation of as many as 60 radialvelocity standards may not be unreasonable - but to check them all as thoroughly as I have suggested needs a major effort of cooperation. A photometric system may require hundreds of standards (McClure 1976).

Sometimes, however, the number of possible standards is strictly limited by the "state of the art" and by the patience of the artist. For example, there are excellent high-resolution atlases of the spectra of three stars - the Sun (Minnaert et al. 1940, Delbouille, et al. 1973), Arcturus (Griffin 1968) and Procyon (Griffin and Griffin 1979). The spectra of these stars are not standards in the full sense that I have defined - that of Arcturus is known to be peculiar - but the very 
existence of these atlases has resulted in these three spectra being used for reference and comparison. Even in these days, however, there are not many stars that can be observed at the requisite degree of resolution over a wide spectral range. This fact circumscribed the choice of stars to be observed in this way. Whatever theoretical principles we may lay down, the stars we select as standards are to some extent dictated by what our instruments can do, and, even more strictly, by what we ourselves are prepared to do. Not many of us are willing to take the time or the care to produce a high-resolution spectral atlas. Nevertheless, the selection of any kind of standard requires that kind of patient and not very glamorous work, if it is to be done properly.

The final requirement for standard stars, that they be suitable for testing instruments, also helps to determine their choice. As I have already suggested, this requirement rules out the adoption of early-type stars as velocity standards. Similarly one would hardly choose a WolfRayet star - with its anomalous, strong, broad emission lines - as a photometric standard. When the inclusion, in a system, of some stars not suitable for testing is unavoidable (as with McClure's late-type giants) then those stars should not be regarded as, in the fullest sense, standard stars.

\section{DIFFERENT SETS OF STANDARDS}

The Universe (or even the Galaxy) has not, unfortunately, been so arranged that there are 20 or 30 stars, distributed around the sky, that can be adopted as standards for everything. Each set of standards must be adopted specifically for its purpose. The specific requirements of one purpose may contradict those of another. For example, although a rapidly rotating star may be unsuitable as a velocity standard, some of them must be included in any set of standards for the measurement of rotational velocities. Similarly, known binaries (of any kind) should be avoided for radial-velocity standards, but McAlister and Hartkopf (1983) have proposed a set of standards for speckle interferometry that are necessarily binaries. Each new method of observation is 1ikely to create its own need for standards.

There may also be a need for hierarchies of standards. Each application in which standard stars are used will require certain primary or fundamental standards, that should be adopted only after the most careful investigation. Many people will adopt provisional standards of their own, hoping that eventually their system of measurement can be tied into a fundamental one. For example, Griffin (1969) found it convenient to adopt four personal velocity standards - which, by now, are probably better observed than many of the I.A.U. standards.

Similarly, Glushneva (1983) has selected a number of standard stars for a spectrophotometric catalogue. Stars chosen in this way form a second level of standards. They usually have been chosen more or less carefully by someone who has a specific purpose in mind; they may or may not prove, later, to have a wider application. When fundamental standards have finally been chosen, these secondary standards can be related to them. At the lowest level are "comparison" stars - I am using the term 
more widely than its narrow photometric sense. These are stars chosen for specific differential measurements - often with convenience or availability in mind, rather than the accuracy with which their relevant properties are known. Sooner or later, these stars must themselves be investigated.

The determination of radial-velocities by means of the objective prism requires many standards, ideally one in every field, but certainly some hundreds (Fehrenbach 1967). Obviously, it is impracticable to choose and test so large a number of primary standards. Fortunately, it is unnecessary to do so because of the relatively low precision of which the method is capable. Secondary or tertiary standards would be sufficient and could eventually be chosen. Again, I emphasize that standards - or comparison stars - are chosen with a particular instrument or method in mind. Another example of radial-velocity work for which a hierarchical ordering of standards is desirable has already been mentioned - cross-correlation. For this method an appreciable number of reference stars is required, so that fairly small intervals of spectral type can each have at least one. There is not yet any general agreement on which stars should be used.

\section{FUNDAMENTAL CALIBRATION}

The observant reader will have noticed that I have so far ignored one important question: how do we measure, for the standard stars themselves, the quantities for which those stars were chosen to be standards? There is an element of circular argument in the use of standard stars. The question has been asked, in recent years: is the Sun really of spectral type G2V? One answer (Garrison and Zimmerman 1983) might be summarized "the Sun is G2V because G2V is what the Sun is". If we rephrase the question to ask if the Sun has an effective temperature and luminosity similar to those of other stars classified as G2V, the answer is not so trivial and will, I expect, be debated at this symposium. I shall not join that debate in this review; I cite the issue only to draw attention to a possible danger in the use of standard stars. A similar problem is raised by standard-velocity stars: how do we know their velocities? Historically, of course, spectrograms of the sky or of solar-system objects were used. The radial velocities of these objects can be calculated independently of any spectroscopic evidence, from the kinematics of the solar system. Thus lines and wavelengths in the spectrum that give the "right" answer can be selected and applied with some confidence to solar-type stars. The system can be extended to other spectral types by observing stars in moving clusters or in visual binaries of which one component is a solar-type star (Petrie 1962). Extended objects such as the sky or planets, however, illuminate the collimator differently from the way in which a stellar point-source does. Moreover, if the spectrograph slit is not correctly placed across the image of the disk of a planet, the planet's rotation may affect the measured velocity. These objections are of more importance now that high-dispersion observations of high internal precision are possible. Fortunately, the brighter asteroids are observable at 
dispersions of $5 \AA \mathrm{mm}^{-1}$ to $10 \AA \mathrm{mm}^{-1}$ and have the same advantages as the major planets without, excepting possibly Ceres, the disadvantages. Preliminary results obtained from Victoria do suggest that the zeropoint of the standard-star system may be slightly in error.

The circle of argument inherent in the use of standard stars can, then, be broken if a fundamental calibration is made. Not everyone need make it - after all, standard stars are used precisely because they are more convenient - but the fundamental calibration must sometimes be made. If we have all agreed on the same set of standards, at least an improved calibration is unlikely to do more than modify our zero-point. Blaauw (1963) has discussed - in connection with the Mount Wilson spectroscopic parallaxes - how a random error in the calibration of an observed quantity against some theoretical parameter can produce a systematic error in both the scale and the zero-point of the derived relation. Observational errors in the eye estimates of luminositysensitive line ratios combined with "cosmic scatter" amongst stars of similar spectral characteristics lead to the deduction of an erroneous relation between the estimates and the absolute magnitudes, and to an underestimate of the true scatter of individual points about the relation. Even if we could achieve infinite internal precision in our observations, cosmic scatter will still work in this way and, indeed, will become proprtionately more important as our precision increases. Lutz and Kelker (1973) have also drawn attention to systematic errors in luminosity calibrations based on trigonometrical parallaxes that can arise from the random errors in the parallaxes. Earlier treatments of both matters can be found in Trumpler and Weaver (1953).

A similar effect is found in radial-velocity standards, for rather different reasons. Dravins (1975) showed that observed velocities of solar-type stars, in particular, may differ by up to $0.5 \mathrm{~km} \mathrm{~s}^{-1}$ from the centers of mass of the stars. Granulation on the solar surface is evidence of (roughly) radial currents, rising and falling in the region where spectral lines are formed. At first sight, this might be supposed to increase only the random error in our knowledge of stellar velocities. Dravins points out, however, that the hotter, brighter rising currents will make a greater contribution to the observed spectrum than will the darker, falling currents. Observed velocities, therefore, will tend to be systematically too negative. The amount of error will differ unpredictably from star to star, particularly since the temperature difference between dark and bright areas will, largely through blending and asymmetry, cause shifts in the rest positions of individual lines. Thus, regardless of the internal precision of the method of measurement, there is not only a possible systematic error, but a minimum random error in the determinations of radial velocities of solar-type (i.e. standard!) stars. Whether or not the possible zero-point difference between asteroids and stars, which we have found at Victoria with a dispersion of $6.5 \AA \mathrm{mm}^{-1}$, is related to this systematic error is not yet clear. The existence of a minimum random error, however, is of increasing importance as our potential internal precision improves. The minimum value was not large enough to be important for the old singleprism spectrographs - even in measures of solar-type stars. It is now comparable in size with the external scatter we find in our 
"conventional" high-dispersion observations. Methods are now being developed and used for which an internal precision of the order of 0.01 $\mathrm{km} \mathrm{s} \mathrm{s}^{-1}$ is claimed (Campbel1 1983, Serkowski 1976). Dravins has shown that no star is stable enough in observed velocity (since the effects of granulation will certainly change with time) to be used as a standard for measurements of such precision. Asteroids merely reflect the solar spectrum, so their spectra will contain the same sources of uncertainty. The present century, in particular its last few decades, has been a time of significant increases in the internal precision of virtually all forms of measurement. In astronomy, the trend seems likely to continue for some time, and it may actually be accelerated by the increasing use of space instruments. An example is provided by the great increase in the precision of positional measurements expected from HIPPARCOS. I have pointed out that the difficulties of choosing standard stars increase with the level of precision attainable. I began the preparation of this paper convinced that the careful selection and testing of standard stars for many purposes would be of considerable importance for some time to come. Now, I am forced to ask myself if it is the most appropriate method of calibration at the highest levels of precision. Although it has its roots in the late nineteenth century, the use of standard stars is particularly characteristic of the twentieth. Is it necessarily to be of the same importance in the twenty-first? Calibration of some kind, of course, will be even more important. We must, as I have shown, calibrate our standard stars in some absolute or fundamental way. Difficult and time-consuming though this may be, we shall perhaps find that really high-precision observations should be directly calibrated, without the aid of the intermediate step of standard stars. As I finished this paper, I discovered - by chance - that, in the first year of the twentieth century, Belopolsky (1901) reported his attempts to measure, in the laboratory, Doppler shifts corresponding to velocities of the same order as those of stars. I believe the paper is largely forgotten and probably will not be referred to by any other participant in this symposium. Perhaps it was just 100 years ahead of its time.

\section{ACKNOWLEDGMENTS}

Many of the ideas presented in this review were developed after a conversation with $\mathrm{Dr}$. J. Andersen of Copenhagen University Observatory. I am also grateful to my colleagues Drs. G. Hill, R.D. McClure and C.D. Scarfe for comments on an earlier draft of this paper.

\section{REFERENCES}

Andersen, J. and Nordström, B. 1983, Astron. Astrophys. Supp. 53, 287 .

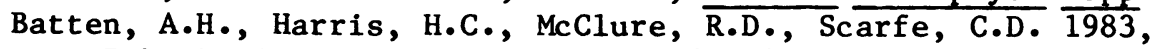
Pub. Dominion Ast rophys. Obs. 16, 143.

Belopolsky, A. 1901, Astrophys. J. 13, 15.

Blaauw, A. 1963, in Basic Astronomical Data, ed. K.Aa. Strand, 
Univ. of Chicago Press, p. 383.

Campbe11, B. 1983, Pub. Astron. Soc. Pacific 95, 577.

Delbouille, L., Neven, L. and Roland, G. 1973, Photometric Atlas of the Solar Spectrum from $\lambda 3000$ to $\lambda 10000$, Liège.

Dravins, D. 1975, Astron. Ast rophys - 43, 45.

Fehrenbach, Ch. 1967, in The Determination of Radial Velocities and their Applications, eds. A.H. Batten and J.F. Heard, Academic Press, London and New York, p. 149.

Garrison, R.F. and Zimmerman, L. 1983, J. Roy. Astr. Soc. Canada, 77 ; 78.

Glushneva, I.N. 1983, Soviet Astron. 27, 326.

Griffin, R.F. 1968, A Photometric Atlas of the Spectrum of Arcturus, Cambridge Philosophical Society.

- 1969, Mon. Not. Roy. Astron. Soc. 145, 163.

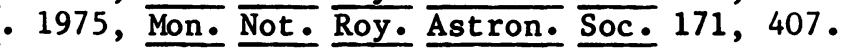

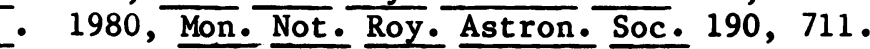

Griffin, R.F. and Griffin, R.E.M. 1979, A Photometric Atlas of the Spectrum of Procyon $\lambda \lambda 3140-7470 \AA$.

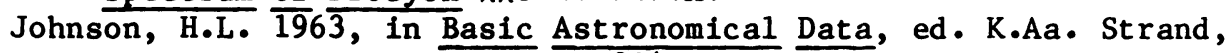
Univ. of Chicago Press, p. 204.

Johnson, H.L. and Morgan, W.W. 1951, Astrophys. J. 114, 522. - 1953, Astrophys. J. 117, 313.

Keenan, P.C. and McNeil, R.C. 1976, An Atlas of Stellar Spectra of the Cooler Stars, Ohio State University Press.

Lutz, T.E. and Kelker, D.H. 1973, Pub. Astron. Soc. Pacific 85, 573.

McAlister, H.A. and Hartkopf, W.I. 1983, Pub. Astron. Soc. Pacific 95, 778 .

McClure, R.D. 1976, Astron. J. 81, 182. - 1983, Pub. Astron. Soc. Pacific 95, 201.

Minnaert, M.G.J., Mulders, G.F. W. and Houtgast, J. 1940, $\underline{\text { A Photometric }}$ Atlas of the Solar Spectrum, Amsterdam: Schnabel.

Morgan, W.W., Keenan, P.C. and Kellman, E. 1943, An Atlas of Stellar Spectra, Univ. of Chicago Press.

Pearce, J.A. 1957, Trans. Int. Astron. Un. 9, ed. P.Th. Oosterhof, Cambridge Univ. Press, p. 441 .

Petrie, R.M. 1962, in Astronomical Techniques, ed.W.A. Hiltner, Univ. of Chicago Press, p. 63.

Petrie, R.M. and Pearce, J.A. 1961, Pub. Dominion Astrophys. Obs. 12,1 .

Serkowski, K. 1976, Icarus 27, 13.

Trumpler, R.J. and Weaver, H.F. 1953, Statistical Astronomy, Berkeley, Univ. of California Press, $\$ 1.51$ and p. 369. 


\section{DISCUSSION}

BESSELL: Now that many radial velocities are measured by cross correlation techniques in the visual and near infrared regions, there is a great need for non solar-like reference stars, such as $M$ stars and carbon stars, where $\mathrm{TiO}$ and $\mathrm{CNO}$ or $\mathrm{C}_{2}$ bands can be used. In particular, systematic velocity differences due to varying band saturation could be explored given a grid of reference velocity stars (based on metal lines) but with a range of TiO and/or CN strengths.

BATTEN: Your comment underlines my points about the need for different types and levels of standards for different purposes. The whole question of reference stars for cross-correlation needs careful consideration. Perhaps this should be done at the radial velocity colloquium next October.

BOHLIN: Could you please describe in more detail the problems especially associated with using $B$ stars as radial velocity standards with a precision of $1 \mathrm{~km} / \mathrm{sec}$ ? What are the prospects of obtaining standards with this precision for the hotter stars that could be used in the UV down to $1150 \AA$ \&?

BATTEN: The problems are the difficulty of measuring rotationally broadened spectral lines to that precision and the fact that no one knows if the velocities of B-type stars are constant to that degree.

KREEOWSKI: One of the criteria that standard stars should fulfill is the lack of reddening effects in their spectra. This is the difficult problem in OB stars where unreddened objects are very scarce. Two of the early-type stars proposed as standards on a list distributed before the symposium, HD 3360 and 160762, were observed by the ANS satellite. These photometric data show clearly the presence of reddening effects.

JASCHEK: Dr. Batten mentioned that the first condition to be fulfilled by a standard star is that it be constant in the parameter concerned. I hope much more attention can be paid to stars which are chosen to be standards for one parameter, but which in fact are variable in another one.

GRIFFIN: In mentioning my four radial velocity reference stars Dr. Batten generously refrained from embarrassing me by reporting the discovery by one of his own colleagues at Victoria, Dr. McClure, that one of the four stars is itself a spectroscopic binary. This is an object lesson to me. The amplitude of the variation is about equal to the measuring error and although I have made more than a thousand observations of the star I have usually compared it with stars which are themselves variable so the variation of the reference star has not been apparent. The higher precision of the Victoria radial velocity spectrometer and the use of a laboratory reference instead of other standard stars has enabled the variation to be discovered and now that :t has been pointed out it is in fact easily traceable in the residuals 
of the stars with which I have compared it.

BATTEN: It is an object lesson to us all: even 1000 observations may not be enough. 